\title{
miR-491-5p suppresses cell growth and invasion by targeting Notch3 in nasopharyngeal carcinoma
}

\author{
QI ZHANG ${ }^{*}$, QIANG LI* ${ }^{*}$ TAO XU, HUI JIANG and LIN-GEN XU \\ Department of ENT, Jinshan Hospital Affiliated to Fudan University, Shanghai 201508, P.R. China
}

Received December 9, 2015; Accepted January 12, 2016

DOI: $10.3892 /$ or.2016.4713

\begin{abstract}
MicroRNAs (miRNAs) have critical roles in the progression of nasopharyngeal carcinoma (NPC), a highly invasive and metastatic cancer that is widely prevalent in Southern China. miR-491-5p has been implicated in multiple types of cancer; however, its biological role and underlying mechanism in NPC have not been fully explored. In the present study, we found that miR-491-5p was downregulated in NPC tissues and cell lines compared with the corresponding normal counterparts. Overexpression of miR-491-5p significantly inhibited cell proliferation, migration and invasion in vitro and suppressed tumor growth in vivo. Using miRNA target prediction algorithms and reporter assays, we showed that miR-491-5p suppressed Notch3 expression both at the mRNA and protein level through directly targeting the $3^{\prime}$ untranslated region (3'-UTR) of Notch3 mRNA. Overexpression of Notch3 significantly reversed the tumor-suppressive effects of miR-491-5p. Taken together, the present study reveals a mechanistic link between miR-491-5p and Notch3 in the pathogenesis of NPC and that miR-491-5p has potential as a therapeutic target for NPC.
\end{abstract}

\section{Introduction}

Nasopharyngeal carcinoma (NPC) is a non-lymphomatous squamous cell carcinoma arising from epithelial cells located in the nasopharynx (1). NPC is a rare disease in most parts of the world and the incidence is $0.2-0.5$ cases $/ 100,000$ people in the USA, but is high in Southern China and Southeast Asia (2-5). NPC responds well to radiotherapy and adjuvant chemotherapy; however, the 5-year overall survival rate is still

Correspondence to: Dr Lin-Gen Xu, Department of ENT, Jinshan Hospital Affiliated to Fudan University, 1508 Longhang Road, Shanghai 201508, P.R. China

E-mail: hrre306@126.com

*Contributed equally

Key words: miR-491-5p, nasopharyngeal carcinoma, Notch3, proliferation, invasion
$70 \%(6-8)$. Therefore, it is important to elucidate the pathogenesis of NPC for discovering new therapeutic approaches.

MicroRNAs (miRNAs) are a class of short single-stranded non-coding RNAs, 22-25 nucleotides, repressing protein translation through directly binding to the 3'-untranslated region (3'-UTR) of target mRNAs, leading to mRNA degradation or translational suppression. miRNAs participate in the regulation of a variety of basic biological processes such as development, cellular differentiation, proliferation, apoptosis, invasion and metabolism (9-11). A large number of studies have demonstrated that the gain or loss of function of miRNAs contributes to cancer development through silencing of tumor suppressor genes or upregulation of oncogenes, including in NPC, such as miR-21, miR-506, miR-223 and miR-142-3p (12-15). miR-491-5p is deregulated and acts as a tumor suppressor in many tumors, including cervical and breast cancer, oral squamous cell and ovarian carcinoma (16-19). However, the function of miR-491-5p in NPC pathogenesis, as well as the molecular mechanisms by which miR-491-5p exerts its function and modulates the malignant phenotypes of NPC cells, is not fully understood.

Notch genes (Notch 1-4) encode single-pass, heterodimeric transmembrane receptors that serve as receptors for the Jagged and $\delta$-like ligands $(20,21)$. The Notch pathway has pivotal roles in cellular processes such as proliferation, invasion, differentiation and development $(22,23)$. Notably, Notch signaling plays key roles in the progression of cancer, and some Notch inhibitors have shown therapeutic efficacy in preclinical studies $(22,24)$.

In the present study, we aimed to explore the potential regulatory mechanisms of miR-491-5p in NPC. We showed for the first time that miR-491-5p is downregulated in NPC, and its overexpression could suppress proliferation and invasion of NPC cells through suppression of Notch3 expression.

\section{Materials and methods}

Cell culture and clinical sample preparation. Four human NPC cell lines (C666-1, CNE1, CNE2 and SUNE-1) were cultured in RPMI-1640 medium (Invitrogen, Carlsbad, CA, USA) supplemented with $10 \%$ fetal bovine serum (FBS). The normal nasopharyngeal epithelia cell line NP69 was cultured in keratinocyte/serum-free medium (Invitrogen) supplemented with bovine pituitary extract (BD Biosciences, San Jose, CA, USA) (25). HEK293T cells were cultured in Dulbecco's 
modified Eagle's medium (DMEM) at $37^{\circ} \mathrm{C}$ in a humidified atmosphere with $5 \% \mathrm{CO}_{2}$. Twenty NPC tissue samples and 10 normal nasopharyngeal epithelial specimens were frozen in liquid nitrogen and stored at $-80^{\circ} \mathrm{C}$ until further use. Informed consent was obtained from all patients and the present study was approved by the Ethics Committee of the Jinshan Hospital Affiliated to Fudan University.

$R N A$ preparation and quantitative reverse transcriptase polymerase chain reaction ( $q R T-P C R$ ) analysis. Total RNA was extracted from cell lines and tissues using the TRIzol (Invitrogen). RNA was reverse transcribed to cDNA using PrimeScript First Strand cDNA synthesis kit, qRT-PCR was performed by SYBR Premix Ex Taq (both from Takara, Dalian, China). $\beta$-actin or U6 was used for normalization. The relative expression levels of the gene of interest were calculated using the $2^{-\Delta \Delta C t}$ method. All reactions were carried out in triplicate and all experiments were performed on three independent occasions.

Oligonucleotides and plasmid transfection. miR-491-5p mimic and negative control were synthesized by RiboBio (Guangzhou, China). The Notch3-3'-UTR wild-type (WT) sequence was amplified and cloned into psiCHECK-2 luciferase reporter vector (Promega, Madison, WI, USA). Mutant 3/-UTR of Notch3 containing two mutated sequences within the miR-491-5p target sites was generated. For Notch3 overexpression, the full-length open reading frame of Notch3 without 3'-UTR was subcloned into pcDNA3.1 vector (Invitrogen). Cells were transfected with miR-491-5p mimic or plasmid using Lipofectamine 2000 reagent (Invitrogen).

Western blot analysis. Cells were lysed in RIPA buffer and the protein concentrations were evaluated using BCA protein assay kit (both from Beyotime, Nantong, China). Total protein was prepared and separated by $10 \%$ SDS-PAGE, then transferred onto polyvinylidene difluoride (PVDF) membranes and blocked with 5\% non-fat milk. The target proteins were detected according to the standard methods with the following primary antibodies: anti-Notch3 antibody and goat antiperoxidase-conjugated secondary antibody (both from Santa Cruz Biotechnology, Santa Cruz, CA, USA). An anti- $\beta$-actin antibody (Sigma, St. Louis, MO, USA) was used as the loading control.

Lentivirus production and transduction. miR-491-5p precursor sequences were amplified and cloned into the lentiviral vector pCDH-CMV-MCS-EF1-copGFP (System Biosciences, Mountain View, CA, USA). A lentiviral vector that expressed GFP alone was used as a control. Virus particles were harvested $48 \mathrm{~h}$ after pCDH-CMV-miR-491-5p transfection with the packaging plasmids psPAX2 and pMD into 293T cells using Lipofectamine 2000 reagent. Target cells were infected with recombinant lentivirus-transducing units plus $8 \mathrm{mg} / \mathrm{ml}$ Polybrene (Sigma).

Cell proliferation assay. Cell viability was detected using the 3-(4,5-dimethyl-2-thiazolyl)-2,5-diphenyl-2H-tetrazolium bromide (MTT) assay. Cells were seeded in 96-well plates at $2 \times 10^{3}$ cells/well and incubated for 24, 48, 72 and $96 \mathrm{~h}$. Next,
$5 \mu 1$ MTT solution was added to each well and then terminated by $200 \mu \mathrm{l}$ dimethyl sulphoxide (DMSO). The viability of cells was detected and measured at $490 \mathrm{~nm}$ using a microplate reader (Bio-Rad, Richmond, CA, USA). All experiments were repeated three times.

Cell migration and invasion assays. We used a Transwell chamber assay (24-well plates, 8-mm pore size; Corning, Corning, NY, USA) coated without or with Matrigel (BD Biosciences), respectively, on the upper surface of the membrane to determine the effect of miR-491-5p on migration and invasion in vitro. Cells $\left(2.5 \times 10^{4}\right)$ were placed in the top chambers in triplicate. For the invasion assay, the upper chamber was coated with Matrigel. Cells on the upper surface of the membrane were removed after $24 \mathrm{~h}$ incubation, and cells on the undersurface were fixed, stained with $0.1 \%$ crystal violet and counted under a light microscope. The Transwell migration assay was performed in the same way as the invasion assay, but without the Matrigel coating.

Cell cycle analysis. Cells at 80-90\% confluence were transfected with miR-491-5p or negative control. After $48 \mathrm{~h}$, cells were collected by trypsinization, washed twice in phosphatebuffered saline (PBS) and fixed in 70\% ethanol. These cells were incubated with DNA-binding dye propidium iodide and RNase for $30 \mathrm{~min}$ at $37^{\circ} \mathrm{C}$. Cells were analyzed on a flow cytometer (FACSCalibur; BD Biosciences).

Luciferase reporter assay. For dual luciferase assays, cells were transiently transfected with appropriate reporter plasmid and miRNA using Lipofectamine 2000. After 48 h, cells were collected and luciferase activity was measured using a Dual Luciferase Reporter Assay kit (Promega).

In vivo tumor growth model. For tumor growth assay, $10^{6}$ SUNE-1 cells stably overexpressing miR-491-5p or negative control were suspended in $200 \mu \mathrm{l}$ PBS, and then subcutaneously injected into nude mice. Four weeks later, the mice were sacrificed, tumors were dissected and weighed, and tumor volume was calculated every three days. The volumes were calculated as follows: volume $=\left(\mathrm{D} \mathrm{x} \mathrm{d}^{2}\right) / 2$, where $\mathrm{D}$ was the longest diameter and $d$ the shortest diameter. All experiments involving animals were undertaken in accordance with the Animal Experimentation Ethics Committee Guide for the Care and Use of Laboratory Animals, with the approval of the Institutional Animal Care and Use Committee of Fudan University.

Statistical analysis. All statistical analyses were performed using SPSS version 16.0 software (SPSS, Inc., Chicago, IL, USA) and are expressed as mean \pm SD. Differences between groups were determined by Student's t-test or by two-way analysis of variance. $\mathrm{P}<0.05$ was considered significant.

\section{Results}

miR-491-5p is downregulated in NPC cell lines and tissues. To examine the role of miR-491-5p in NPC, the expression level of miR-491-5p was detected by qRT-PCR in NPC cell lines and tissues. As shown in Fig. 1A, miR-491-5p was expressed 

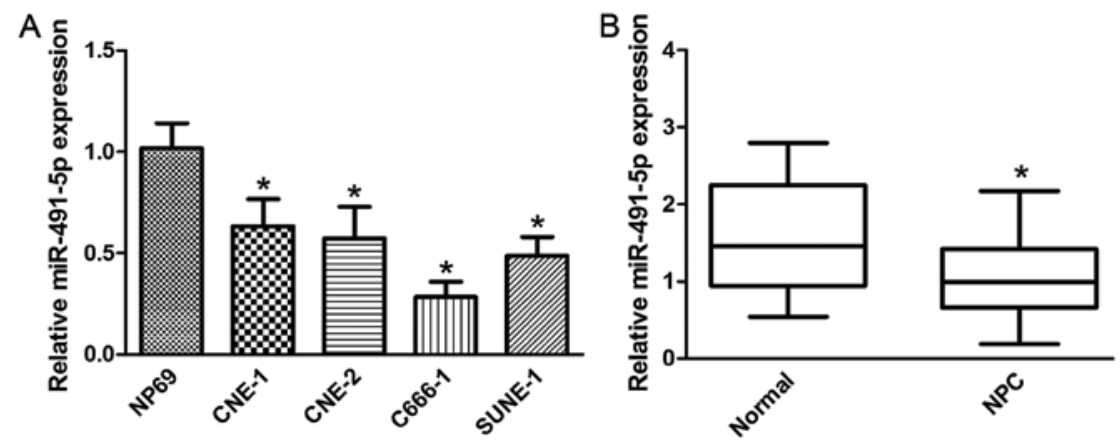

Figure 1. miR-491-5p is downregulated in NPC cell lines and tissues. (A) Expression of miR-491-5p in four NPC cell lines and the normal nasopharyngeal epithelia cell line (NP69) was measured by qRT-PCR. (B) Expression of miR-491-5p in 20 NPC tissues and 10 normal nasopharyngeal epithelial tissues; "P<0.05.

A

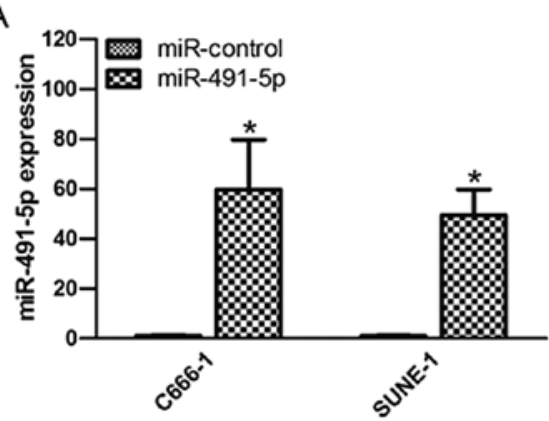

$\circ$

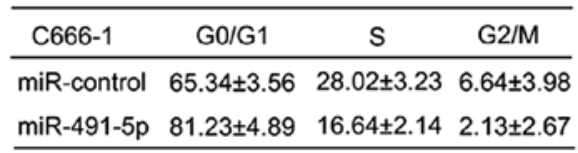

\begin{tabular}{cccc}
\hline SUNE-1 & G0/G1 & S & G2/M \\
\hline miR-control & $67.34 \pm 2.35$ & $25.27 \pm 5.46$ & $6.39 \pm 6.46$ \\
miR-491-5p & $80.22 \pm 2.15$ & $12.74 \pm 4.65$ & $7.04 \pm 4.94$ \\
\hline
\end{tabular}

D

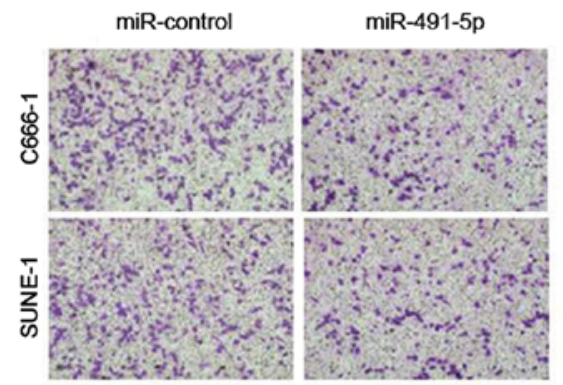

E

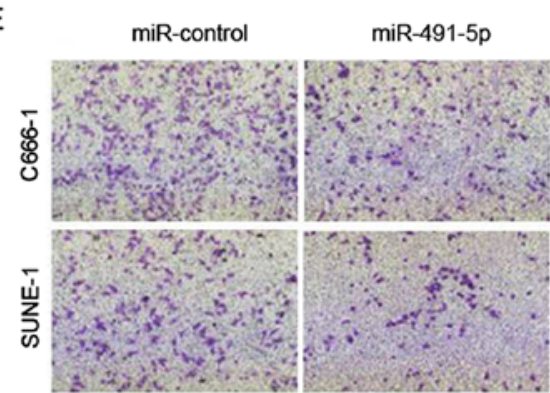

B
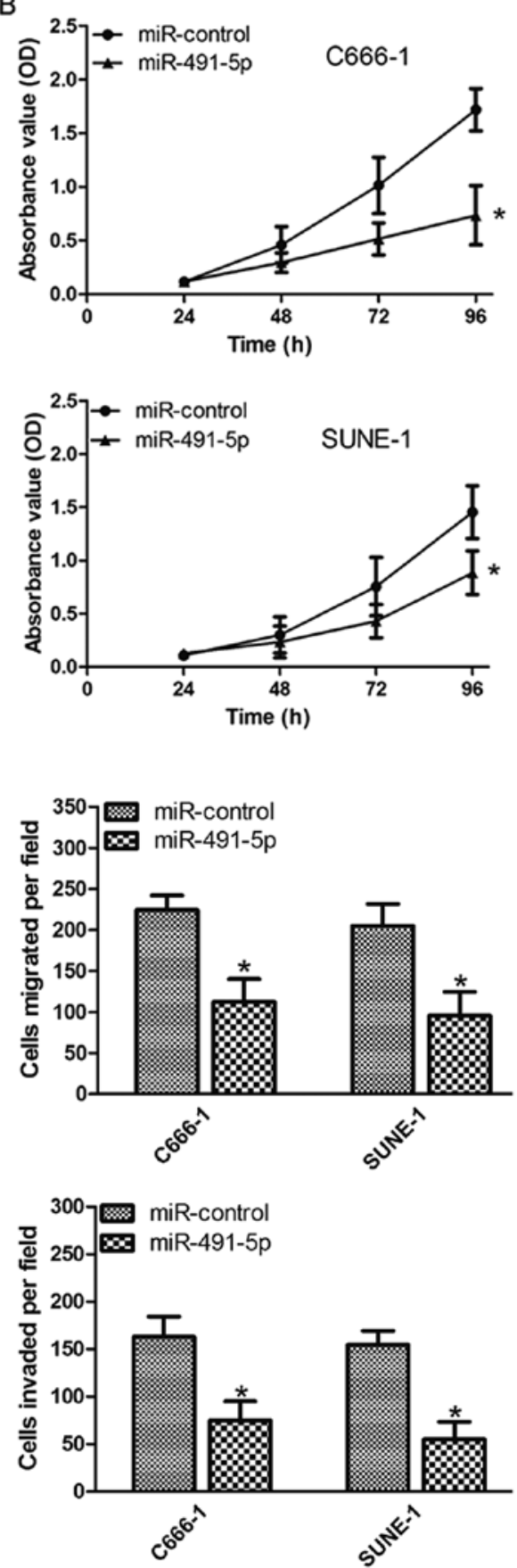

Figure 2. miR-491-5p inhibits NPC cell proliferation, migration and invasion and induces cell cycle arrest in vitro. (A) qRT-PCR analysis of miR-491-5p expression in C666-1 and SUNE-1 cells transfected with miR-491-5p or negative control. (B) MTT assay was performed to test viability of the indicated cells. Representative results of migration (C), invasive ability (D) and cell cycle (E) in the indicated cells; ${ }^{*} \mathrm{P}<0.05$. 
A

Notch3 position $248-254$

W' 3'-UTR 5'...CUGGGACCUCCUUCUUCCCCACU...3'

miR-491-5p 3' GGAGUACCUUCCCAAGGGGGUGA 5'

mutant 3'-UTR 5'...CUGGGACCUCCUUCUAGGGGUGA...3'

Notch3 position 630-636

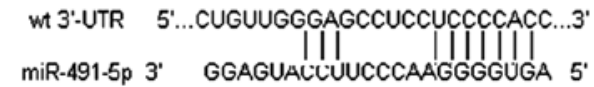

mutant 3'-UTR 5'...GUGUUGCCUGCCUCCAGGGGUGC...3'

C

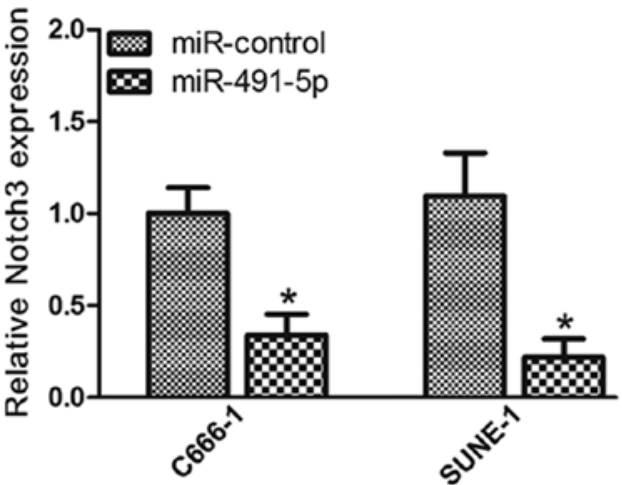

B

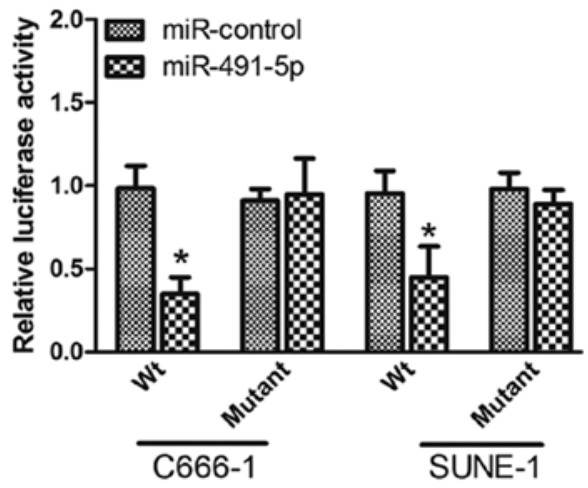

D

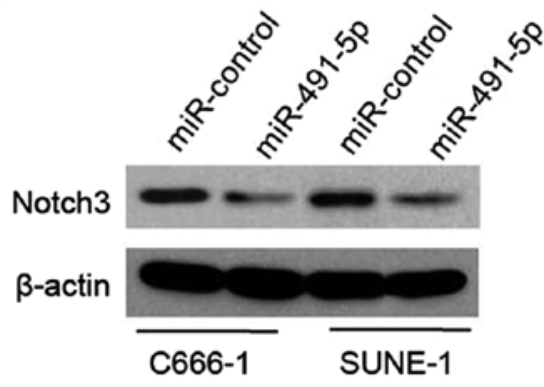

Figure 3. Notch3 is a direct target of miR-491-5p. (A) Schematic diagram of the putative binding sites of miR-491-5p in the Notch3 3'-UTR. The mutant sequences Notch3 3'-UTR used in the luciferase reporter constructs are shown. (B) Relative luciferase activity in C666-1 and SUNE-1 cells co-transfected with wild-type (wt) or mutant Notch3 3'-UTR reporter genes and miR-491-5p mimic or negative control. The mRNA (C) and protein expression (D) levels of Notch3 in C666-1 and SUNE-1 cells transfected with miR-491-5p mimic or negative control were determined by qRT-PCR and western blotting; ${ }^{*} \mathrm{P}<0.05$.

at markedly lower levels in all NPC cell lines tested, compared to the normal nasopharyngeal epithelial cell line NP69. miR-491-5p was also downregulated in NPC tissues compared with normal nasopharyngeal epithelial tissues (Fig. 1B).

Effects of miR-491-5p overexpression on NPC cell proliferation, migration and invasion. To investigate the effects of miR-491-5p on NPC cell growth, C666-1 and SUNE-1 cells were transfected with miR-491-5p mimic or negative control (Fig. 2A). Using the MTT assay, we found that overexpression of miR-491-5p significantly inhibited cell proliferation compared with the negative control (Fig. 2B). We also tested the effects of miR-491-5p on the cell cycle progression by flow cytometric analysis. Compared with the miR-control group, miR-491-5p overexpression increased the percentage of cells in $\mathrm{G} 1 / \mathrm{G} 0$ phases and decreased the percentage of cells in $\mathrm{S}$ phase (Fig. 2C). Transwell migration and invasion assays showed that miR-491-5p overexpression inhibited cell migration and invasion compared to the negative control (Fig. 2D and E). These results demonstrate that miR-491-5p suppresses several malignancy parameters in human NPC cells.

Notch3 is a target of $m i R-491-5 p$. The miRNA target analysis tools miRanda and TargetScan were used to explore potential targets of miR-491-5p and Notch3 was selected due to its positive roles in cancer cell proliferation and invasion. Luciferase reporter assay was performed to determine whether Notch3 was a direct target of miR-491-5p. We first cloned the WT or mutant miR-491-5p target sequences of the Notch3 3'-UTR into luciferase reporter vectors (Fig. 3A). After co-transfection with miR-491-5p mimic, the luciferase activity of the WT 3'-UTR reporter gene reduced significantly, whereas the activity of the mutant reporter gene was not affected (Fig. 3B), confirming that miR-491-5p can bind to the Notch3 3'-UTR. In addition, we analyzed the level of Notch 3 expression in C666-1 and SUNE-1 cells transfected with miR-491-5p mimic or miR-control by qRT-PCR and western blotting. miR-491-5p overexpression significantly reduced the Notch3 expression at both the mRNA and protein levels (Fig. 3C and D). These data indicate that Notch 3 was directly and negatively regulated by miR-491-5p.

Notch3 attenuates the suppressive effect of miR-491-5p. Given that miR-491-5p could suppress cell proliferation, migration and invasion as well as inhibit the expression of Notch3 through regulating Notch3 3'-UTR, we predicted that ectopic expression of Notch 3 may reverse the suppressive effects of miR-491-5p. Notch3 ORF lacking 3'-UTR was co-transfected with miR-491-5p in C666-1 and SUNE-1 cells, and western blotting validated expression of Notch3 (Fig. 4A). As shown in Fig. 4B-D, restoration of Notch3 partially rescued proliferative and invasive ability impaired by miR-491-5p. These data indicate that Notch 3 is critically involved in mediating the tumor-inhibitory function of miR-491-5p.

Antitumor effects of miR-491-5p on NPC xenografts. To explore the function of miR-491-5p on tumor growth, we 
A

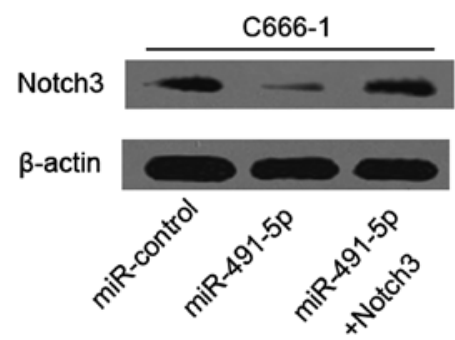

B
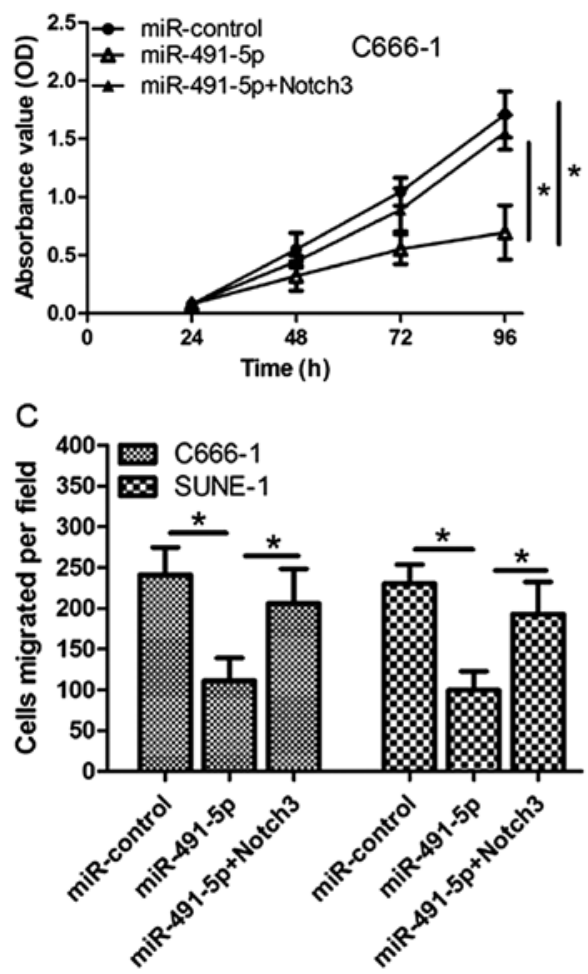

SUNE-1
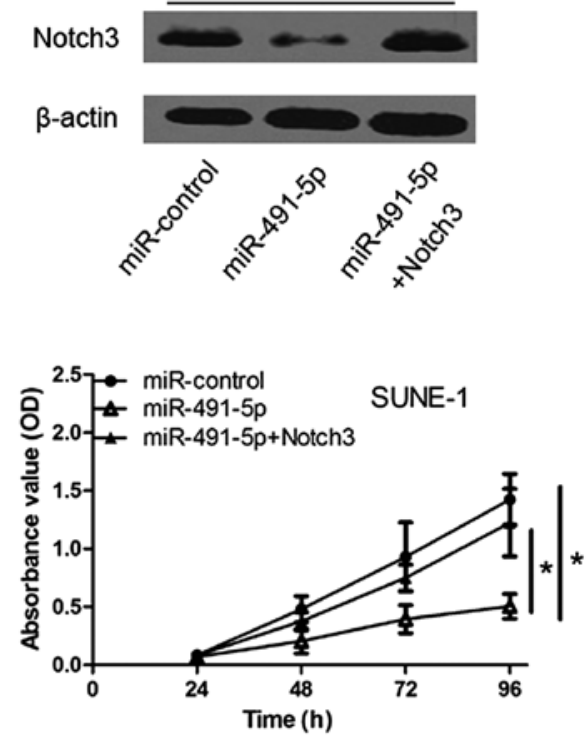

D

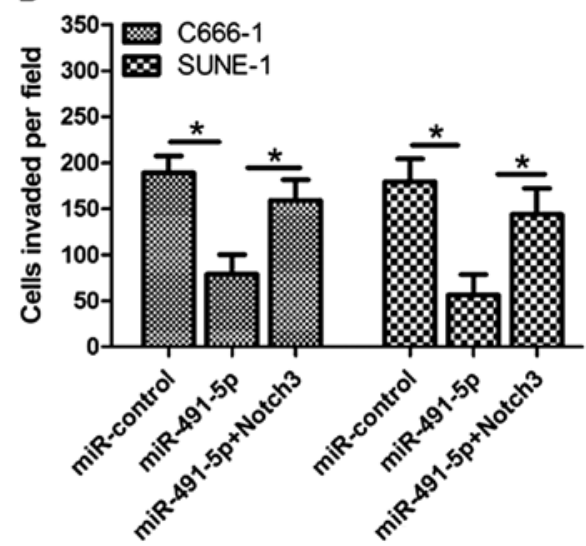

Figure 4. Overexpression of Notch3 attenuates the tumor-suppressive effects of miR-491-5p. (A) Expression of Notch3 was measured using western blotting in the indicated cells. Notch3 counteracted the suppression of proliferation (B), migration (C) and invasion (D) of C666-1 and SUNE-1 cells caused by miR-491-5p compared with that observed in control cells. Cell proliferation was detected using MTT assay, and cell migration and invasion were determined using the Transwell assays; ${ }^{*} \mathrm{P}<0.05$.

constructed a SUNE-1 cell line stably overexpressing miR-491-5p by lentivirus-mediated transduction. These cells were injected subcutaneously into the flanks of nude mice, and tumor growth was measured until sacrifice of the mice after 28 days. The tumors of the LV-miR-491-5p group had smaller volumes and sizes than the control tumors (Fig. 5A and B). The average tumor weight was also significantly lower in the LV-miR-491-5p group (Fig. 5C). Furthermore, we examined cell proliferation of NPC and the expression of miR-491-5p and Notch3 in the subcutaneous tissues. The results revealed that LV-miR-491-5p increased tumor miR-491-5p levels, reduced the expression of Notch3 protein, and suppressed expression of cell proliferation marker Ki67 compared with negative control (Fig. 5D-F). These data indicate that miR-491-5p can significantly inhibit NPC cell growth, and it acts as a negative regulator in NPC.

\section{Discussion}

It is now widely accepted that miRNAs contribute to cancer development by acting as oncogenes or tumor suppressor genes. Previous studies have shown that miR-491-5p is dysregulated in various cancers and its potential function has also been partly explored in several studies (16-18). For example, Zhao et al reported that miR-491-5p suppressed cervical cancer cell proliferation, migration and invasion, induced cell apoptosis and suppressed tumor growth in a mouse model (16). Zeng et al found that miR-491-5p functions as a tumor suppressor by targeting JMJD2B in estrogen-receptor$\alpha$-positive breast cancer (17). Huang et al showed that miR-491-5p and GIT1 serve as modulators and biomarkers for oral squamous cell carcinoma invasion and metastasis (18). These consistent results suggest that miR-491-5p functions as a tumor suppressor and affects cell biological behavior in cancer. However, to date, no functional evidence of miR-491-5p in NPC has been documented. Therefore, further extensive investigations are required to identify miR-419-5p that is involved in the cell growth of NPC.

In the present study, we found that miR-491-5p expression was markedly downregulated in human NPC cells and tissues. Increased expression of miR-491-5p inhibited cell proliferation, migration and invasion and induced cell cycle arrest. We 
A

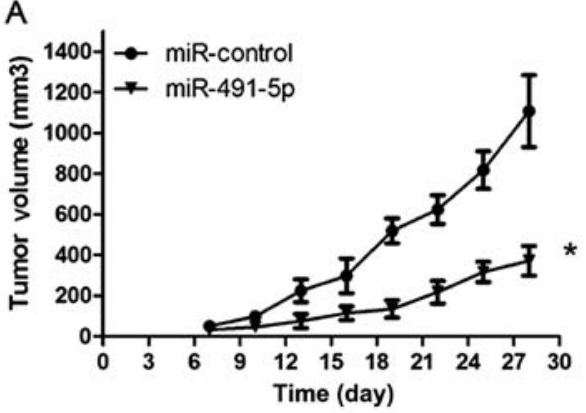

C

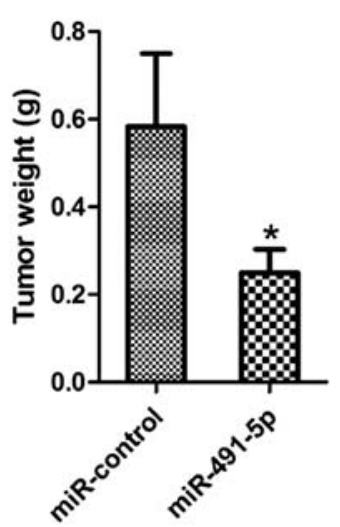

$\mathrm{F}$
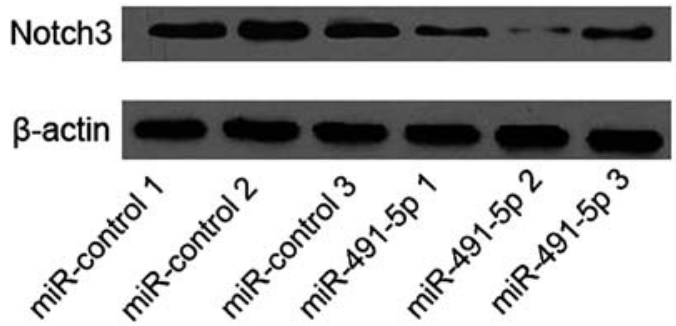

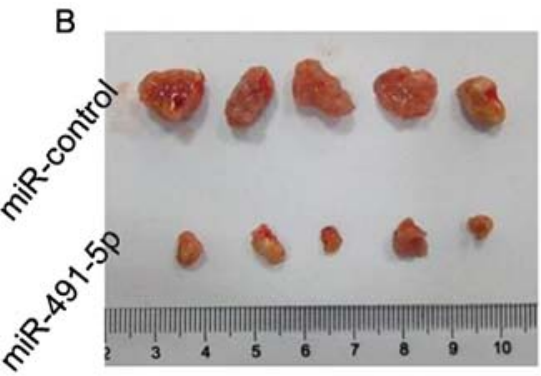

$E$
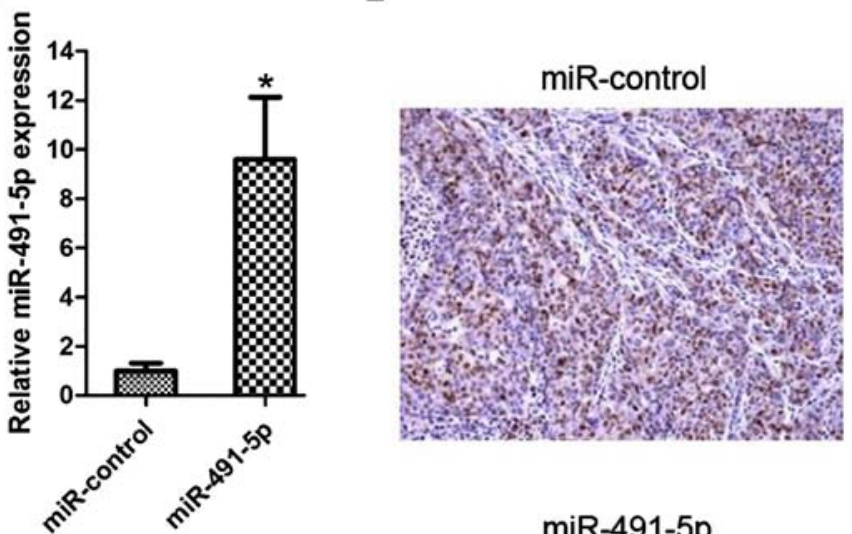

\section{$\operatorname{miR}-491-5 p$}

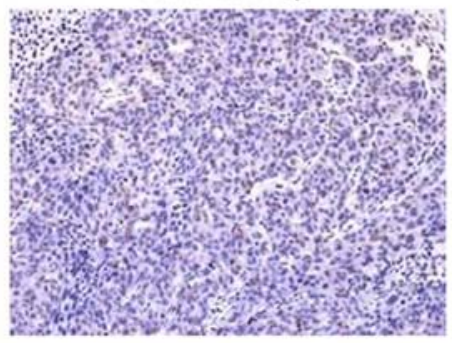

Figure 5. miR-491-5p suppresses NPC xenograft tumor growth in vivo. SUNE-1 cells infected with LV-miR-491-5p or LV-miR-control were subcutaneously injected into the nude mice. (A) The growth curves of tumor volumes. (B) Representative image of tumors formed. (C) Tumor weight. (D) qRT-PCR analysis of the expression levels of miR-491-5p in the miR-491-5p overexpressing tumors and control tumors. (E) Representative immunohistochemical staining of Ki67-stained cells in indicated tumors. (F) Tumor levels of Notch3 proteins measured using western blotting; ${ }^{*} \mathrm{P}<0.05$.

also explored the role of miR-491-5p in vivo using a xenograft mouse model and found that tumor growth was suppressed when miR-491-5p was overexpressed. These data suggest a tumor-inhibitory role of miR-491-5p in NPC cells both in vitro and in vivo.

The mechanism of the effect of miR-491-5p on the development of NPC was also investigated in the present study. Notch3 was predicted to be a theoretical target gene of miR-491-5p. We found that miR-491-5p bound to the 3'-UTR of Notch3 mRNA and repressed its expression. Further studies indicated that overexpression of Notch3 reversed the tumor suppressive effects of miR-491-5p on NPC cells. All these data suggest that Notch3 is a direct and functional target of miR-491-5p in NPC cells.

Notch3 is one of the four different Notch proteins (Notch1-4), which have emerged as important players in cancer therapy, and which have been found to affect diverse processes, such as proliferation, invasion and migration. For instance, Notch3 was upregulated in ovarian cancer and its inhibitor reduced viability, migration and angiogenesis, and increased apoptosis (26). Jaskula-Sztul et al indicated that Notch3 suppressed medullary thyroid cancer cell proliferation and induced apoptosis (27). Alqudah et al showed that Notch3 plays a major role in glioma cell proliferation, migration, invasion and apoptosis (28). Notch3 is regulated by miRNAs in many cancers. Wang et al found that miR-206 inhibited tumor proliferation and migration involving the downregulation of Notch3 in colorectal cancer (29). Furukawa et al suggested that the miR-1/Notch3 pathway is important for colorectal tumor cell migration and may be a promising molecular target for the treatment of colorectal tumors (30). Previous studies reported that Notch3 is upregulated in NPC cell lines, and targeting Notch3 signaling may serve as a potential therapeutic approach in patients with Epstein-Barr-virus-associated NPC (31). Thus, it is particularly important for the exploration of the regulatory mechanism of Notch3 in NPC development, 
and the present study showed for the first time that Notch3 was a direct downstream target gene of miR-491-5p, affecting NPC development.

In conclusion, we demonstrated that miR-491-5p is downregulated in NPC and may function as a tumor suppressor by directly targeting Notch3. To the best of our knowledge, this is the first study to demonstrate that the miR-491-5p/Notch3 axis regulates the proliferation, migration and invasion of NPC cells. These findings provide a better understanding of the pathogenesis and development of NPC and may have important implications for future therapy of NPC.

\section{Acknowledgements}

The present study was supported by the Key Specialty Construction Project of Medical Health System in The Fourth Cycle, Jinshan, Shanghai (2012-26).

\section{References}

1. Zhang SX, Qiu QH, Chen WB, Liang $\mathrm{CH}$ and Huang B: Celecoxib enhances radiosensitivity via induction of $\mathrm{G}_{2}-\mathrm{M}$ phase arrest and apoptosis in nasopharyngeal carcinoma. Cell Physiol Biochem 33: 1484-1497, 2014.

2. Kamran SC, Riaz N and Lee N: Nasopharyngeal carcinoma. Surg Oncol Clin N Am 24: 547-561, 2015.

3. Lu J, Zhao FP, Peng Z, Zhang MW, Lin SX, Liang BJ, Zhang B, Liu X, Wang L, Li G, et al: EZH2 promotes angiogenesis through inhibition of miR-1/Endothelin-1 axis in nasopharyngeal carcinoma. Oncotarget 5: 11319-11332, 2014.

4. Chen LX, Zhu LY, Jacob TJ and Wang LW: Roles of volumeactivated $\mathrm{Cl}^{-}$currents and regulatory volume decrease in the cell cycle and proliferation in nasopharyngeal carcinoma cells. Cell Prolif 40: 253-267, 2007.

5. Luo Z, Dai Y, Zhang L, Jiang C, Li Z, Yang J, McCarthy JB, She X, Zhang $\mathrm{W}$, Ma J, et al: miR-18a promotes malignant progression by impairing microRNA biogenesis in nasopharyngeal carcinoma. Carcinogenesis 34: 415-425, 2013.

6. Zhang T, Sun Q, Liu T, Chen J, Du S, Ren C, Liao G and Yuan Y: MiR-451 increases radiosensitivity of nasopharyngeal carcinoma cells by targeting ras-related protein 14 (RAB14). Tumour Biol 35: 12593-12599, 2014.

7. Huang GL, Chen ML, Li YZ, Lu Y, Pu XX, He YX, Tang SY, Che H, Zou Y, Ding C, et al: Association of miR-146a gene polymorphism with risk of nasopharyngeal carcinoma in the central-southern Chinese population. J Hum Genet 59: 141-144, 2014.

8. Huang GL, Lu Y, Pu XX, He YX, Chen ML, Li YZ, Tang SY, Che $\mathrm{H}$ and $\mathrm{He} \mathrm{Z}$ : Association study between miR-149 gene polymorphism and nasopharyngeal carcinoma. Biomed Rep 1: 599-603, 2013.

9. Bartel DP: MicroRNAs: Target recognition and regulatory functions. Cell 136: 215-233, 2009.

10. Kloosterman WP and Plasterk RH: The diverse functions of microRNAs in animal development and disease. Dev Cell 11: 441-450, 2006.

11. He L and Hannon GJ: MicroRNAs: Small RNAs with a big role in gene regulation. Nat Rev Genet 5: 522-531, 2004.

12. Li Y, Yan L, Zhang W, Wang $\mathrm{H}$, Chen W, Hu N and $\mathrm{Ou} \mathrm{H}$ : miR-21 inhibitor suppresses proliferation and migration of nasopharyngeal carcinoma cells through down-regulation of BCL2 expression. Int J Clin Exp Pathol 7: 3478-3487, 2014.

13. Zhang Z, Ma J, Luan G, Kang L, Su Y, He Y and Luan F: MiR-506 suppresses tumor proliferation and invasion by targeting FOXQ1 in nasopharyngeal carcinoma. PLoS One 10: e0122851, 2015.
14. Yang W, Lan X, Li D, Li T and Lu S: MiR-223 targeting MAFB suppresses proliferation and migration of nasopharyngeal carcinoma cells. BMC Cancer 15: 461, 2015.

15. Qi X, Li J, Zhou C, Lv C and Tian M: MiR-142-3p suppresses SOCS6 expression and promotes cell proliferation in nasopharyngeal carcinoma. Cell Physiol Biochem 36: 1743-1752, 2015.

16. Zhao Q,Zhai YX, Liu HQ, Shi YA and Li XB: MicroRNA-491-5p suppresses cervical cancer cell growth by targeting hTERT. Oncol Rep 34: 979-986, 2015.

17. Zeng H, Yiling C, Wenting Y, XuQun H, ChuanYi Z and Hui L: miR-491-5p functions as a tumor suppressor by targeting JMJD2B in ER $\alpha$-positive breast cancer. FEBS Lett 589: 812-821, 2015.

18. Huang WC, Chan SH, Jang TH, Chang JW, Ko YC, Yen TC, Chiang SL, Chiang WF, Shieh TY, Liao CT, et al: miRNA-491-5p and GIT1 serve as modulators and biomarkers for oral squamous cell carcinoma invasion and metastasis. Cancer Res 74: 751-764, 2014.

19. Denoyelle C, Lambert B, Meryet-Figuière M, Vigneron N, Brotin E, Lecerf C, Abeilard E, Giffard F, Louis $\mathrm{MH}$, Gauduchon $\mathrm{P}$, et al: miR-491-5p-induced apoptosis in ovarian carcinoma depends on the direct inhibition of both BCL- $\mathrm{X}_{\mathrm{L}}$ and EGFR leading to BIM activation. Cell Death Dis 5: e1445, 2014.

20. Artavanis-Tsakonas S, Rand MD and Lake RJ: Notch signaling: Cell fate control and signal integration in development. Science 284: 770-776, 1999.

21. Baron M: An overview of the Notch signalling pathway. Semin Cell Dev Biol 14: 113-119, 2003.

22. Fre S, Huyghe M, Mourikis P, Robine S, Louvard D and Artavanis-Tsakonas S: Notch signals control the fate of immature progenitor cells in the intestine. Nature 435: 964-968, 2005.

23. Ranganathan P, Weaver KL and Capobianco AJ: Notch signalling in solid tumours: A little bit of everything but not all the time. Nat Rev Cancer 11: 338-351, 2011.

24. Yuan X, Wu H, Xu H, Xiong H, Chu Q, Yu S, Wu GS and Wu K: Notch signaling: An emerging therapeutic target for cancer treatment. Cancer Lett 369: 20-27, 2015.

25. Lu J, Luo H, Liu X, Peng Y, Zhang B, Wang L, Xu X, Peng X, Li G, Tian W, et al: miR-9 targets CXCR4 and functions as a potential tumor suppressor in nasopharyngeal carcinoma. Carcinogenesis 35: 554-563, 2014.

26. Kang H, Jeong JY, Song JY, Kim TH, Kim G, Huh JH, Kwon AY, Jung SG and An HJ: Notch3-specific inhibition using siRNA knockdown or GSI sensitizes paclitaxel-resistant ovarian cancer cells. Mol Carcinog: Jul 24, 2015 (Epub ahead of print). doi: $10.1002 / \mathrm{mc} .22363$.

27. Jaskula-Sztul R, Eide J, Tesfazghi S, Dammalapati A, Harrison AD, Yu XM, Scheinebeck C, Winston-McPherson G, Kupcho KR, Robers MB, et al: Tumor-suppressor role of Notch3 in medullary thyroid carcinoma revealed by genetic and pharmacological induction. Mol Cancer Ther 14: 499-512, 2015.

28. Alqudah MA, Agarwal S, Al-Keilani MS, Sibenaller ZA, Ryken TC and Assem M: NOTCH3 is a prognostic factor that promotes glioma cell proliferation, migration and invasion via activation of CCND1 and EGFR. PLoS One 8: e77299, 2013.

29. Wang XW, Xi XQ, Wu J, Wan YY, Hui HX and Cao XF: MicroRNA-206 attenuates tumor proliferation and migration involving the downregulation of $\mathrm{NOTCH} 3$ in colorectal cancer. Oncol Rep 33: 1402-1410, 2015.

30. Furukawa S, Kawasaki Y, Miyamoto M, Hiyoshi M, Kitayama J and Akiyama T: The miR-1-NOTCH3-Asef pathway is important for colorectal tumor cell migration. PLoS One 8: e80609, 2013.

31. Man CH, Wei-Man Lun S, Wai-Ying Hui J, To KF, Choy KW, Wing-Hung Chan A, Chow C, Tin-Yun Chung G, Tsao SW, Tak-Chun Yip T, et al: Inhibition of NOTCH3 signalling significantly enhances sensitivity to cisplatin in EBV-associated nasopharyngeal carcinoma. J Pathol 226: 471-481, 2012. 